\title{
Quasi-stationarity of scalar turbulent mixing statistics in a non-symmetric
}

\section{case}

\author{
Luis Valiño, ${ }^{1}$ Juan Hierro, ${ }^{2, \text { a) }}{ }^{\text {Radu Mustata, }}{ }^{1}$ and César Dopazo ${ }^{1}$ \\ ${ }^{1)}$ LIFTEC, CSIC-Universidad de Zaragoza, María de Luna 10, 50018, Zaragoza, SPAIN ${ }^{\mathrm{b})}$ \\ ${ }^{2)}$ Centro Universitario de la Defensa, Carr. de Huesca, s/n, 50090 Zaragoza, SPAIN
}

(Dated: 9 March 2021)

The existence of an asymptotic shape at large times of the probability density function (PDF) of a non-reacting scalar, mixed by a solenoidal turbulent velocity field and molecular diffusive transport, was investigated by Sinai and Yakhot ${ }^{1}$. The quasi-stationarity of the mixing statistics along the time evolution by Valiño et al. ${ }^{7}$ was an extension to symmetric scalar pdfs; analytic solutions for the scalar fluctuation dissipation rates, conditional upon the scalar value, and the pdf were obtained. This manuscript examines the generalization of the latter results to asymmetric scalar pdfs and further scrutinizes underlying mechanisms for a quasi-stationary statistics.

\section{INTRODUCTION}

Non-Gaussian statistics of scalar fields undergoing turbulent mixing was investigated ${ }^{1-4}$ about thirty years ago. Ted and his students ${ }^{5,6}$ showed that the asymptotic scalar PDFs display a Gaussian-like behavior only near the mean value and that the conditional average scalar fluctuation dissipation rates are different from those of a scalar Gaussian field. The scalar PDF shape for asymptotically large times ${ }^{1}$, was extended to include the symmetric PDF time evolution; the underlying assumption of even moment statistical quasi-stationarity allowed obtaining analytic expressions for the average scalar fluctuation dissipation rates, conditioned on the scalar value, and the PDFs at various mixing times ${ }^{7}$. The time-invariance of the mixture fraction PDF was a necessary condition ${ }^{8}$ for the constancy of the scalar mean value normalized with its variance ${ }^{9}$.

It was apparent in the 1990 decade that existing mixing model predictions of various scalar moments did not agree with their asymptotic values obtained by direct numerical simulations (DNS $)^{10}$. A set of new models, including the Johnson-Edgeworth Translation ${ }^{11,12}$, of which the amplitude mapping closure ${ }^{13}$ is a particular case, was specialized to investigate the mixing of a binary symmetric scalar in homogeneous turbulence; while the PDFs obtained with the various tested models compared well with DNS results, none of them accurately predicted neither the conditional diffusion nor the conditional fluctuation dissipation rate of the scalar field ${ }^{14}$. This predictive failure was associated to the models not accounting for the migration of scalar lower and upper bounds.

The self-similar passive scalar normalized PDFs at finite times in homogeneous turbulence were obtained via DNS ${ }^{15}$, finding that the PDF central regions displayed power laws ${ }^{1}$; however, while Gaussian shapes were apparent for large Peclet numbers, small values of that dimensionless parameter yielded PDFs, which resemble stretched or pure exponential functions.

The objective of this manuscript is to extend previous results ${ }^{7}$ to a diffusive scalar with an initially asymmetric PDF

\footnotetext{
a)Electronic mail: hierro@unizar.es

b)Electronic mail: 1.valino@csic.es
}

in a statistically homogeneous turbulent velocity field. The mathematical formulation of the mixing of a statistically homogeneous scalar field is revisited, the implications of the statistical quasi-stationarity are analyzed in depth and a discussion on the limitations of approximating the conditional dissipation rate by low order polynomials are discussed. Comment on the underlying mechanisms of the scalar statistical quasistationarity and further extensions are also presented.

\section{MATHEMATICAL FOUNDATIONS}

The scalar conservation equation is

$$
\frac{\partial Y}{\partial t}+v_{j} \frac{\partial Y}{\partial x_{j}}=\alpha \nabla^{2} Y
$$

$v_{j}$ is the jth component of the statistically homogeneous turbulent velocity vector field, which is assumed solenoidal, $\partial v_{j} / \partial x_{j}=0$, and zero-mean, $\left\langle v_{j}\right\rangle=0 . \alpha$ stands for the molecular diffusivity of the scalar field $Y(x, t)$. The angular brackets represent statistical or ensemble average.

$Y(x, t)$ is conventionally decomposed into its mean, $\langle Y\rangle(t)$, and its fluctuations, $y(x, t)$, namely, $Y(x, t)=\langle Y\rangle(t)+y(x, t)$.

Time evolution equations for various central moments of $y(x, t)$ can be readily obtained. For example, the scalar fluctuation variance is defined by, $\sigma_{y}^{2}(t)=\left\langle y^{2}\right\rangle$.

The normalized scalar variable $X(x, t)=y(x, t) / \sigma_{y}(t)$ obeys the conservation equation:

$$
\frac{\partial X}{\partial t}+v_{j} \frac{\partial X}{\partial x_{j}}=\alpha \nabla^{2} X+\left\langle\varepsilon_{X}\right\rangle X
$$

$\left\langle\varepsilon_{X}\right\rangle=\left\langle\alpha|\nabla X|^{2}\right\rangle=\frac{\left\langle\alpha|\nabla y|^{2}\right\rangle}{\sigma_{y}^{2}(t)}$ is the dissipation rate of scalar and $\left\langle\varepsilon_{X}\right\rangle^{-1}$ is a characteristic mixing time for both $Y$ and $X$. Note that $\langle X(x, t)\rangle=0$ and $\left\langle[X(x, t)]^{2}\right\rangle=\frac{\left\langle[y(x, t)]^{2}\right\rangle}{\sigma_{y}^{2}(t)}=1$. The last term in eq. (2) acts like a linear chemical source term generating $X$. 


\section{A. Scalar quasi-stationarity}

Even and odd central moments of $X$ obey, respectively, the time evolution equations:

$$
\begin{aligned}
\frac{d\left\langle X^{2 n}\right\rangle}{d t^{*}} & =-2 n(2 n-1) \frac{\left\langle X^{2 n-2} \varepsilon_{X}\right\rangle}{\left\langle\varepsilon_{X}\right\rangle}+2 n\left\langle X^{2 n}\right\rangle \\
\frac{d\left\langle X^{2 n+1}\right\rangle}{d t^{*}} & =-2 n(2 n+1) \frac{\left\langle X^{2 n-1} \varepsilon_{X}\right\rangle}{\left\langle\varepsilon_{X}\right\rangle}+(2 n+1)\left\langle X^{2 n+1}\right\rangle
\end{aligned}
$$

$d t^{*}=\left\langle\varepsilon_{X}\right\rangle d t$ is a stretched dimensionless differential time.

From general eqs.(3), low order moment equations may be readily obtained,

$$
\begin{aligned}
& \frac{d\left\langle X^{2}\right\rangle}{d t}=0 \\
& \frac{d\left\langle X^{3}\right\rangle}{d t}=-6 \frac{\left\langle X \varepsilon_{X}\right\rangle}{\left\langle\varepsilon_{X}\right\rangle}+3\left\langle X^{3}\right\rangle \\
& \frac{d\left\langle X^{4}\right\rangle}{d t}=-12 \frac{\left\langle X^{2} \varepsilon_{X}\right\rangle}{\left\langle\varepsilon_{X}\right\rangle}+4\left\langle X^{4}\right\rangle .
\end{aligned}
$$

Scalar statistical quasi-stationarity would imply:

$$
\begin{aligned}
2 \frac{\left\langle X \varepsilon_{X}\right\rangle}{\left\langle\varepsilon_{X}\right\rangle} & \approx\left\langle X^{3}\right\rangle=S \\
3 \frac{\left\langle X^{2} \varepsilon_{X}\right\rangle}{\left\langle\varepsilon_{X}\right\rangle} & \approx\left\langle X^{4}\right\rangle=F \\
n \frac{\left\langle X^{n-1} \varepsilon_{X}\right\rangle}{\left\langle\varepsilon_{X}\right\rangle} & \approx\left\langle X^{n+1}\right\rangle .
\end{aligned}
$$

The PDF of $X(x, t), P(\chi ; t)=\langle\delta[\chi-X(x, t)]\rangle$, is governed by the alternative transport equations

$$
\begin{aligned}
& \frac{\partial P(\chi ; t)}{\partial t}=\frac{-\partial}{\partial \chi}\left\{\left[D(\chi, t)+\left\langle\varepsilon_{X}\right\rangle \chi\right] P(\chi ; t)\right\} \\
& \frac{\partial P(\chi ; t)}{\partial t}=\frac{-\partial}{\partial \chi}\left\{\frac{\partial E(\chi, t) P(\chi ; t)}{\partial \chi}+\left\langle\varepsilon_{X}\right\rangle \chi P(\chi ; t)\right\}
\end{aligned}
$$

$D(\chi, t)=\left\langle\alpha \nabla^{2} X \mid X(x, t)=\chi\right\rangle \quad$ and $\quad E(\chi, t)=$ $\left\langle\alpha|\nabla X|^{2} \mid X(x, t)=\chi\right\rangle$ are the scalar diffusion and fluctuation dissipation rate, conditioned on the scalar value being equal to $\chi$.

Identification of the probability fluxes within the curly brackets implies:

$$
D(\chi, t) P(\chi ; t)=\frac{\partial}{\partial \chi}[E(\chi, t) P(\chi ; t)] .
$$

Integration from the lower bound, $\chi_{m}$ to $\chi$, yields:

$$
P(\chi, t)=\frac{A}{E(\chi, t)} \exp \left[\int_{\chi_{m}}^{\chi} \frac{D\left(\chi^{\prime}, t\right)}{E\left(\chi^{\prime}, t\right)} d \chi^{\prime}\right]
$$

$A=E\left(\chi_{m}, t\right) P\left(\chi_{m} ; t\right)$ is a normalization constant. $P(\chi, t)$ can be obtained from a knowledge of both $D(\chi, t)$ and $E(\chi, t)$.

According to the definition of homogeneous flow:

$$
\left\langle D(X, t) X^{n}\right\rangle=-n\left\langle X^{n-1} \varepsilon_{X}\right\rangle
$$

under quasi-stationarity

$$
\left\langle D(X, t) X^{n}\right\rangle \approx-\left\langle\varepsilon_{X}\right\rangle\left\langle X^{n+1}\right\rangle
$$

Eq. (13) means that $D(\chi, t) \approx-\left\langle\varepsilon_{X}\right\rangle \chi$ in homogeneous, quasi-stationary situations. This result has been previously obtained $^{21}$.

In fact, this is equivalent to the LMSE model, which approximates the average scalar diffusion, conditional upon a prescribed value of the scalar, as a linear function of the latter, namely,

$$
\left\langle\alpha \nabla^{2} X \mid X(\vec{r}, t)=\chi\right\rangle=D(\chi, t)=-6 \alpha \frac{\chi}{\lambda_{X}^{2}} ;
$$

$\lambda_{X}$ is the Taylor micro-scale for the scalar field and the average scalar dissipation rate can be recast as $\left\langle\varepsilon_{X}\right\rangle=\alpha\left\langle X^{2}\right\rangle / \lambda_{X}^{2}=$ $\alpha / \lambda_{X}^{2}$. The LMSE model is then expressed as $D(\chi, t)=$ $-6\left\langle\varepsilon_{X}\right\rangle \chi$; the factor 6 can be embedded in the definition of $\lambda_{X}$

Quasi-stationarity means that scalar diffusion and dissipation rate may be approximated as if the statistics were stationary, yet some evolution of statistical moments higher than two is allowed. Thus, eqs. (8) and (9) are approximated as if both were identically zero in both sides. From eq. (8), $\left\langle D(X, t) X^{n}\right\rangle+\left\langle\varepsilon_{X}\right\rangle\left\langle X^{n+1}\right\rangle=0$, which is simply a restatement of eq. (13). Eq. (9) leads to a more useful condition:

$$
\begin{array}{r}
\frac{\partial}{\partial \chi}[E(\chi, t) P(\chi, t)]+\left\langle\varepsilon_{X}\right\rangle \chi P(\chi, t)= \\
\left.\frac{\partial}{\partial \chi}[E(\chi, t) P(\chi, t)]\right]_{\chi=\chi_{m}}+\left\langle\varepsilon_{X}\right\rangle \chi_{m} P\left(\chi_{m}, t\right)
\end{array}
$$

Integration of the latter implies:

$$
P(\chi ; t)=\frac{C}{E(\chi, t)} \exp \left[-\left\langle\varepsilon_{X}\right\rangle \int \frac{\chi}{E(\chi, t)} d \chi\right] .
$$

A comparison of eqs.(11) and (14) leads to $D(\chi, t)=$ $-\left\langle\varepsilon_{X}\right\rangle \chi$. It should be noted that an exact expression $D(\chi, t)=$ $-\left\langle\varepsilon_{X}\right\rangle \chi$ means a stationary condition: the shape of the PDF does not change, nor do the scalar statistical moments of order higher than two ${ }^{21}$. Quasi-stationarity, with a modification of scalar statistical moments and PDF's shape, requires that the expression be only approximate.

The quasi-stationary scalar PDF, $P(\chi ; t)$, can be obtained if the conditional scalar fluctuation dissipation rate, $E(\chi, t)$, is known and the closure assumption that $D(\chi, t) \approx-\left\langle\varepsilon_{X}\right\rangle \chi$ is applied. The conditional scalar fluctuation dissipation can be approximated as a polynomial, namely, $E(\chi, t)=$ $\sum_{\alpha=0}^{N} K_{\alpha} \chi^{\alpha}$. A second order expression, with $K_{1}=0$, has been previously assumed for the case of symmetric $P(\chi ; t)^{7}$. Here, a quadratic approximation is used: 


$$
E(\chi, t)=K_{0}+K_{1} \chi+K_{2} \chi^{2}=\left\langle\varepsilon_{X}\right\rangle \frac{1+C_{1} \chi+C_{2} \chi^{2}}{1+C_{2}} .
$$

The formulation with $C_{i}$ instead of $K_{i}$ has been introduced because it corresponds to the straightforward extension of that used in the symmetric case ${ }^{7}$.

The integral in the exponent of eq. (14) can be readily computed:

$$
\left\langle\varepsilon_{X}\right\rangle \int \frac{\chi}{E(\chi, t)} d \chi=\left(1+C_{2}\right) \int \frac{\chi}{1+C_{1} \chi+C_{2} \chi^{2}} d \chi
$$

The computation depends on the roots of $1+C_{1} \chi+C_{2} \chi^{2}$. Its discriminant is $C_{1}^{2}-4 C_{2}$ and various cases are possible:

i. $C_{1}^{2}-4 C_{2}>0$, two real roots, the PDF is bounded to the interval between the roots and its value is

$$
\begin{aligned}
P(\chi ; t)= & \frac{C}{\left\langle\varepsilon_{X}\right\rangle}\left(1+C_{2}\right)\left(1+C_{1} x+C_{2} x^{2}\right)^{-\frac{1+3 C_{2}}{2 C_{2}}} \\
& \left(\frac{-C_{1}+\sqrt{C_{1}^{2}-4 C_{2}}-2 C_{2} \chi}{C_{1}+\sqrt{C_{1}^{2}-4 C_{2}}+2 C_{2} \chi}\right)^{\frac{C_{1}\left(1+C_{2}\right)}{2 C_{2} \sqrt{C_{1}^{2}-4 C_{2}}}}
\end{aligned}
$$

ii. $C_{1}^{2}-4 C_{2}<0$, two complex conjugate roots, the PDF is unbounded and is given by

$$
\begin{aligned}
P(\chi ; t)= & \frac{C}{\left\langle\varepsilon_{X}\right\rangle}\left(1+C_{2}\right)\left(1+C_{1} \chi+C_{2} \chi^{2}\right)^{-\frac{1+3 C_{2}}{2 C_{2}}} \\
& \exp \left(\frac{C_{1}\left(1+C_{2}\right) \tan ^{-1}\left(\frac{C_{1}+2 C_{2} \chi}{\sqrt{-C_{1}^{2}+4 C_{2}}}\right)}{C_{2} \sqrt{-C_{1}^{2}+4 C_{2}}}\right)
\end{aligned}
$$

iii. $C_{1}^{2}-4 C_{2}=0$, two real roots with the same value, the $\mathrm{PDF}$ is unbounded and is given by

$$
\begin{aligned}
P(\chi ; t)= & \frac{C}{\left\langle\varepsilon_{X}\right\rangle} 2^{1+\frac{4}{C_{1}^{2}}}\left(4+C_{1}^{2}\right)\left(2+C_{1} \chi\right)^{-3-\frac{4}{C_{1}^{2}}} \\
& \exp \left(-\frac{2\left(4+C_{1}^{2}\right)}{C_{1}^{2}\left(2+C_{1} \chi\right)}\right)
\end{aligned}
$$

In the situation where $S=0, C_{1}=C_{2}=0$, the Gaussian solution of the symmetric case ${ }^{7}$ is recovered.

In all the previous cases

$$
\int P(\chi ; t) d \chi=1
$$

The values of $C_{1}$ and $C_{2}$ are obtained from the condition that the PDF has a given value of skewness, $\left\langle X^{3}\right\rangle=S$, and kurtosis, $\left\langle X^{4}\right\rangle=F$. Therefore,

$$
\begin{aligned}
& C_{1}=\frac{(3+F) S}{4 F-3 S^{2}} \\
& C_{2}=-\frac{6-2 F+3 S^{2}}{4 F-3 S^{2}} .
\end{aligned}
$$

\section{B. Scalar dissipation quasi-stationarity}

A non-Gaussian behavior of PDFs of scalar and velocity gradients has been associated with the internal intermittency of those fields ${ }^{16-20}$. Mixing can be characterized either by the destruction of scalar fluctuations or by the annihilation of scalar gradient fluctuations. The conservation equation for the instantaneous scalar dissipation rate, $\varepsilon_{X}(x, t)=\alpha|\nabla X|^{2}=$ $\alpha|\nabla Y|^{2} / \sigma_{y}^{2}(t)$, can be readily obtained:

$$
\begin{aligned}
\frac{\partial \varepsilon_{X}}{\partial t}+v_{j} \frac{\partial \varepsilon_{X}}{\partial x_{j}}= & -2 a_{N} \varepsilon_{X}+\alpha \nabla^{2} \varepsilon_{X} \\
& -2 \alpha^{2} X_{, i j} X_{, i j}+2\left\langle\varepsilon_{X}\right\rangle \varepsilon_{X} .
\end{aligned}
$$

$a_{N}=n_{i} S_{i j} n_{j}$ is the flow strain rate normal to the iso-scalar surface $X(x, t)=\chi$, where $\chi$ is a constant. The unit vector locally normal to the iso-surface is given by $n_{i}=X_{, i} /|\nabla X|$. The flow strain rate tensor is defined as $S_{i j}=(1 / 2)\left(v_{i, j}+v_{j, i}\right)$. The first term on the right side of the transport equation for $\varepsilon_{X}$ is the enhancement of the scalar dissipation rate by the predominantly compressive (in solenoidal turbulence) flow normal strain rate. The second and third terms are the molecular transport and dissipation of $\varepsilon_{X}$, respectively. The last term results from the normalized definition of $X(x, t)$. Average of eq. (21) leads to:

$$
\frac{d\left\langle\varepsilon_{X}\right\rangle}{d t}=-2\left\langle\varepsilon_{X} a_{N}\right\rangle-2\left\langle\alpha^{2} X_{, i j} X_{, i j}\right\rangle+2\left\langle\varepsilon_{X}\right\rangle^{2} .
$$

The order of magnitude of the first term in eq. (22) is $\left(v^{2} / l_{i n t}^{4} \operatorname{Re}_{l}^{5 / 2}\right.$ for $\alpha<v$ and $\left(v^{2} / l_{i n t}^{4}\right) \mathrm{Sc}^{1 / 2} \mathrm{Re}_{l}^{5 / 2}$ for $\alpha \gg v$; $v$ is the kinematic viscosity, $l_{\text {int }}$ is the turbulent integral length scale, $\mathrm{Sc}=v / \alpha$ is the Schmidt number and $\mathrm{Re}_{l}=u l_{\text {int }} / v$ is the turbulent Reynolds number, with $u$ being the turbulent velocity $\mathrm{rms}^{31}$. The ratio of the last and the first terms of eq. (22) is proportional to $\mathrm{Re}^{-1 / 2}$. In the limit of large Reynolds numbers, the last term in eq. (22) is negligible in comparison with production of $\left\langle\varepsilon_{X}\right\rangle$. Eq. (22) will then describe the approximate asymptotic balance between production and dissipation of $\left\langle\varepsilon_{X}\right\rangle^{31}$.

The scalar dissipation rate PDF, $P_{\varepsilon}(\varepsilon ; t)=$ $\left\langle\delta\left[\varepsilon-\varepsilon_{X}(x, t)\right]\right\rangle$, obeys the two alternative evolution equations:

$$
\frac{\partial P_{\varepsilon}}{\partial t}=\frac{-\partial}{\partial \varepsilon}\left\{\left[\left\langle\alpha \nabla^{2} \varepsilon_{X} \mid \varepsilon_{X}(x, t)=\varepsilon\right\rangle-2\left\langle a_{N} \mid \varepsilon_{X}(x, t)=\varepsilon\right\rangle \varepsilon-2\left\langle\alpha^{2} X,{ }_{i j} X_{, i j} \mid \varepsilon_{X}(x, t)=\varepsilon\right\rangle+2\left\langle\varepsilon_{X}\right\rangle \varepsilon\right] P_{\varepsilon}\right\}
$$




$$
\frac{\partial P_{\varepsilon}}{\partial t}=\frac{-\partial}{\partial \varepsilon}\left\{\frac{\partial}{\partial \varepsilon}\left[\left\langle\alpha\left|\nabla \varepsilon_{X}\right|^{2} \mid \varepsilon_{X}(x, t)=\varepsilon\right\rangle P_{\varepsilon}\right]+\left[-2\left\langle a_{N} \mid \varepsilon_{X}(x, t)=\varepsilon\right\rangle \varepsilon-2\left\langle\alpha^{2} X,,_{i j} X_{, i j} \mid \varepsilon_{X}(x, t)=\varepsilon\right\rangle+2\left\langle\varepsilon_{X}\right\rangle \varepsilon\right] P_{\varepsilon}\right\}
$$

Quasi-stationarity of $P_{\varepsilon}(\varepsilon ; t)$ implies:

$$
P_{\varepsilon}(\varepsilon ; t)=\frac{K}{\left\langle\alpha\left|\nabla \varepsilon_{X}\right|^{2} \mid \varepsilon_{X}(x, t)=\varepsilon\right\rangle} \exp \left(\int \frac{2\left\langle a_{N} \mid \varepsilon_{X}(x, t)=\varepsilon\right\rangle \varepsilon+2\left\langle\alpha^{2} X_{, i j} X_{, i j} \mid \varepsilon_{X}(x, t)=\varepsilon\right\rangle-2\left\langle\varepsilon_{X}\right\rangle \varepsilon}{\left\langle\alpha\left|\nabla \varepsilon_{X}\right|^{2} \mid \varepsilon_{X}(x, t)=\varepsilon\right\rangle} d \varepsilon\right)
$$

$\mathrm{K}$ can be obtained from the normalization condition $\int P_{\varepsilon}(\varepsilon ; t) d \varepsilon=1$.

The various conditional expectations: $\left\langle a_{N} \mid \varepsilon_{X}=\varepsilon\right\rangle$ and $\left\langle\alpha^{2} X,{ }_{i j} X_{, i j} \mid \varepsilon_{X}=\varepsilon\right\rangle$, can be computed from DNS databases. Conditions for an approximately log-normal $P_{\varepsilon}(\varepsilon ; t)$ could be examined.

For asymptotically large values of the Reynolds number eqs. (23), (24) and (25) would simplify as a consequence of the approximate balance between production and dissipation of $\left\langle\varepsilon_{X}\right\rangle$, previously described.

\section{BRIEF DESCRIPTION OF THE NUMERICAL SIMULATION}

A Direct Numerical Simulation (DNS) was used to compute an incompressible, isotropic, homogeneous, statistically stationary turbulent velocity field.

An artificial, incompressible velocity field with zero mean and random fluctuations distributed according to the CeruttiMeneveau turbulent kinetic energy spectrum ${ }^{22,23}$ was chosen as an initial condition of the incompressible Navier-Stokes equations with forcing and periodic boundary conditions

$$
\begin{array}{r}
\frac{\partial u_{i}}{\partial x_{i}}=0 \\
\frac{\partial u_{i}}{\partial t}+u_{j} \frac{\partial u_{i}}{\partial x_{j}}=-\frac{1}{\rho} \frac{\partial P}{\partial x_{i}}+v \frac{\partial^{2} u_{i}}{\partial x_{j} \partial x_{j}}+f_{i}
\end{array}
$$

$f_{i}$ stands for the forcing term.

The numerical integration of Eq.(27) used a pseudospectral numerical code com $^{3,24,25}$ in the spatial domain with a fourth-order Runge-Kutta scheme, with exponential time differencing ${ }^{26,27}$, in the time domain. The reason of using a fourth-order scheme, with exponential integration of linear contributions, instead of a more usual second-order one was that the fields obtained could be used in future chemically active computations which are more demanding.

The computational spatial domain was a cube of edge $2 \pi$ with a $768^{3}$ grid. Convective aliasing was removed with a spherical low-pass filter which sets to zero all spectral nodes corresponding to wave numbers larger than $768 / 3=256$.
TABLE I. Ranges of values used to characterize velocity fields obtained in this DNS: Taylor-based Reynolds number, integral length scale, Taylor length scale, Kolmogorov length scale, kinetic energy dissipation and root mean square velocity.

\begin{tabular}{cccccc}
\hline \hline$R e_{\lambda}$ & $l_{\text {int }}$ & $\lambda$ & $\eta$ & $\langle\varepsilon\rangle$ & $u$ \\
\hline 89,98 & $0.89,0.97$ & $0.30,0.31$ & $0.015,0.016$ & $1.9,2.0$ & $1.5,1.6$ \\
\hline \hline
\end{tabular}

The forcing scheme was that of Eswaran and Pope ${ }^{28}$, with a modification to enforce a zero correlation of the forcing contribution with the forced velocity field ${ }^{29}$. To be more specific, all the Fourier-space nodes with a modulus less than $2 \sqrt{2}$, except the zero node which has no contribution, receive a random, white noise forcing contribution. Its phase is adjusted to enforce incompressibility and perpendicularity to the unforced field, whereas its intensity is such that the Taylor-scale Reynolds number is close to 95.

Velocity fields are characterized by the Taylor-based Reynolds number $\left(R e_{\lambda}\right)$, the integral length scale $\left(l_{\text {int }}\right)$, the length of the edge of the computational domain $(L=2 \pi)$, the viscosity ( $v=0.005$ in this simulation), the Taylor microscale $(\lambda)$, the Kolmogorov microscale $(\eta)$, the kinetic energy dissipation rate $(\langle\varepsilon\rangle)$ and the root mean square velocity $(u)$. Ranges for the values of these variables, along the reported simulation, are represented in table I.

At $t=20 l / u$, an initial velocity field with enough statistical stationarity was chosen. At this point, two initial scalar fields as close as possible to a homogeneously random distribution of 0 and 1 values were added. One of the scalar fields was almost symmetric with mean close to 0.5 whereas the other one was asymmetric with mean close to 0.7 . After that, the reported DNS was started and it run for $t=5 l / u$. Equation (1) was used to represent the evolution of the chemically passive scalar field. The Schmidt number, $S c=v / \alpha$, was 1 .

The time step took a fixed value which led to Courant numbers

$$
C=\frac{\Delta t}{\Delta x / \max \{\|\vec{u}\|\}}
$$

between 0.65 and 0.77 .

Scalar fields are characterized by the diffusivity coefficient $(\alpha=0.005)$; the mean $(\langle Y\rangle)$ : a symmetric scalar with 0.5 mean and an asymmetric one with 0.7 mean; the variance 
TABLE II. Scalar variance and scalar dissipation of the symmetric scalar, mean 0.5 and the asymmetric scalar, mean 0.7 .

\begin{tabular}{lccccc}
\hline \hline & $t_{1}$ & $t_{2}$ & $t_{3}$ & $t_{4}$ & $t_{5}$ \\
\hline$\langle Y\rangle=0.5,\left\langle Y^{2}\right\rangle$ & 0.150 & 0.0690 & 0.0294 & 0.0097 & 0.0030 \\
$\langle Y\rangle=0.5,\left\langle\varepsilon_{Y}\right\rangle$ & 0.0763 & 0.0505 & 0.0261 & 0.0095 & 0.0029 \\
$\langle Y\rangle=0.7,\left\langle Y^{2}\right\rangle$ & 0.124 & 0.0572 & 0.0244 & 0.0078 & 0.0025 \\
$\langle Y\rangle=0.5,\left\langle\varepsilon_{Y}\right\rangle$ & 0.0640 & 0.0418 & 0.0215 & 0.0079 & 0.0022 \\
\hline \hline
\end{tabular}

$\left(\left\langle Y^{2}\right\rangle\right)$ and the scalar fluctuation dissipation rate $\left(\left\langle\alpha Y_{, i} Y_{, i}\right\rangle\right)$.

Results have been analyzed after every large-eddy turn-over time, approximately, up to a maximum of $t u / l=5$. That is to say, a total of five different states. The study of statistical functions: Probability Density Function (PDF), conditional scalar dissipation and conditional scalar diffusion, is realized on normalized fluctuation variables, $X(x, t)=y(x, t) / \sigma_{y}(t)$.

In table II, the values of scalar variance and scalar dissipation are represented for each reported time step. It should be noticed that $\left\langle\varepsilon_{X}\right\rangle=\left\langle\varepsilon_{Y}\right\rangle / \sigma_{y}^{2}$.

\section{RESULTS}

The first point to notice is that asymptotic statistics of the scalar field obtained in the DNS reported does not seem to approach a Gaussian state which should be characterized by a kurtosis close to 3 and a skewness close to 0 , see figure 1. This fact is well known ${ }^{32}$ and very sensitive to the exact distribution of spectral scalar fluctuations in the initial condition $^{5,33}$. This non-Gaussian behavior is interesting from the point of view of modelling since there are some models which work better when an almost asymptotic Gaussian statistics is expected $^{34-36}$.

With regard to the quasi-stationary properties of the scalar fields obtained in the DNS, eqs. (5) and (6) can be considered as a closure assumption which relates joint statistics of a scalar and its dissipation to statistics of the scalar. These equations would be exact if the quasi-stationary assumption were satisfied. A comparison between DNS values of both sides of eqs.(5) and (6) is represented in figure 1. It may be realized that as kurtosis grows, eq. (6) becomes a better approximation (difference between both terms of the equation reduces, evolution of kurtosis in time flattens as a sign of quasi-stationarity); whereas as skewness diminishes, eq. (5) worsens (relative error between both terms of the equation increases). This means that the kurtosis approaches a quasi-stationary state faster than the reduction of initial asymmetry, as represented by the skewness. Nevertheless, it should be noticed that, since absolute values of skewness are smaller than those of kurtosis, absolute errors of the same order imply greater relative errors of skewness. It would be interesting to check the evolution with different initial conditions ${ }^{33}$. One could also ask why this DNS has not been run for a longer time to check the asymptotic behavior of skewness; however, see table II, in the final state the variance of fluctuations is around $2 \%$ of its initial value which corresponds to an almost well-mixed situation.

Figs. 2 and 3 depict the conditional diffusion normalized by
$1 /\left\langle\varepsilon_{X}\right\rangle$ for the symmetric and asymmetric initial distributions of the scalar field. Note that the LMSE is a straight line with slope -1 with such a norm. The agreement of the LMSE with DNS results is reasonable for highly probable scalar values, whereas discrepancies are evident at the extremal scalar values; namely, about the PDF tails. The beginning of the DNS corresponds to a scalar field is an approximate double delta function with a clear departure from the quasi-stationary assumption and LMSE is not a good approximation to the scalar conditional diffusion.

Figs. 4 and 5 depict the conditional dissipation rate normalized by $1 /\left\langle\varepsilon_{X}\right\rangle$ for the symmetric and asymmetric initial distributions of the scalar field. Discrepancies are apparent in the tail probability regions, while the approximation yields reasonable results in highly probable zones near the scalar mean as soon as PDF spikes at the boundaries smoothen. It is worth remarking the shift in the shape of the conditional dissipation from convex to concave for some $t u / l>3.02$.

Figs. 6 and 7 show an excellent agreement between the previous analytical solutions for PDFs in the symmetric and asymmetric cases, respectively, and the DNS results. In the symmetric simulation, $\langle Y\rangle=0.5$, there is a spike in the PDF obtained from the DNS at $t u / l=2.13$ near $\chi=-2$. The reason is that symmetry is not perfectly satisfied by the DNS data.

It must be pointed out that the approximation of the conditional scalar fluctuation dissipation rate, $E(\chi, t)$, by means of a second order polynomial is not unique. In this paper, the alternative to choose a best fit of the implicit asymptotic values of statistical moments of the conditioning variable up to fourth-order has been chosen. This means that a good fit of the PDF is expected, figures 6 and 7, since there is a perfect coincidence of all statistical moments up to fourthorder between the DNS PDF and the modelled PDF. However, the conditional expectation itself is not so well represented, figures 4 and 5, since the implicit assumption that eqs. (5) and (6) hold is not true, see figure 1. Another option would have been to express $E(\chi, t)$ by means of a Quadratic Mean Stochastic Estimation. If there is available information about the joint statistics of a scalar and its gradient: $\left\langle\varepsilon_{X}\right\rangle,\left\langle X \varepsilon_{X}\right\rangle$ and $\left\langle X^{2} \varepsilon_{X}\right\rangle$, one could build a second order approximation to $E(\chi, t)=K_{0}+K_{1} \chi+K_{2} \chi^{2}$, such that

$$
\begin{aligned}
\int E(\chi, t) d \chi & =K_{0}+K_{2}=\left\langle\varepsilon_{X}\right\rangle \\
\int \chi E(\chi, t) d \chi & =K_{1}+K_{2} S=\left\langle X \varepsilon_{X}\right\rangle \\
\int \chi^{2} E(\chi, t) d \chi & =K_{0}+K_{1} S+K_{2} F=\left\langle X^{2} \varepsilon_{X}\right\rangle
\end{aligned}
$$

where use has been made of the properties of the normalized fluctuation variable: $\langle X\rangle=0,\left\langle X^{2}\right\rangle=1,\left\langle X^{3}\right\rangle=S$ and $\left\langle X^{4}\right\rangle=F$. The solution of the system of equations (29) produces an optimal second-order approximation of $E(\chi, t)=$ $K_{0}+K_{1} \chi+K_{2} \chi^{2}$ in a statistical sense: there is more weight towards a good fit in the zones of high probability, whereas more error is allowed in the zones of low probability. However, one cannot expect that such $E(\chi, t)$ corresponds to the right $P(\chi ; t)$, with the right values of skewness and kurtosis after a quasi-stationary reconstruction, eqs. (16), (17) or (18), 


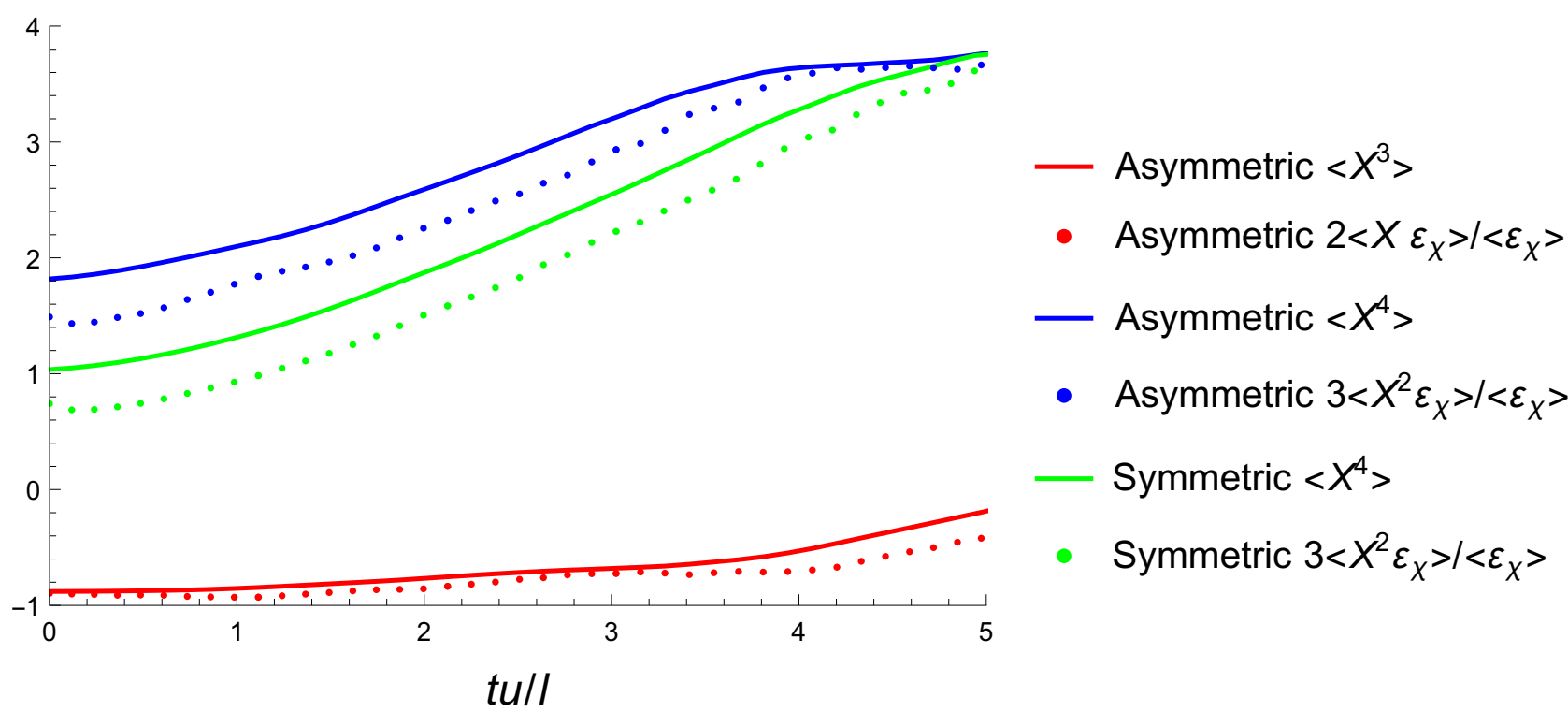

FIG. 1. Skewness, $\left\langle X^{3}\right\rangle$; cross scalar-dissipation, $2\left\langle X \varepsilon_{X}\right\rangle /\left\langle\varepsilon_{X}\right\rangle$; kurtosis, $\left\langle X^{4}\right\rangle$; and cross variance-dissipation, $3\left\langle X^{2} \varepsilon_{X}\right\rangle /\left\langle\varepsilon_{X}\right\rangle$ for the asymmetric and symmetric scalars. DNS results. Skewnes and cross scalar-dissipation is only represented for the asymmetric scalar. Not reported values of skewness for the symmetric scalar were, at least, one order of magnitude less than those obtained for the asymmetric case.

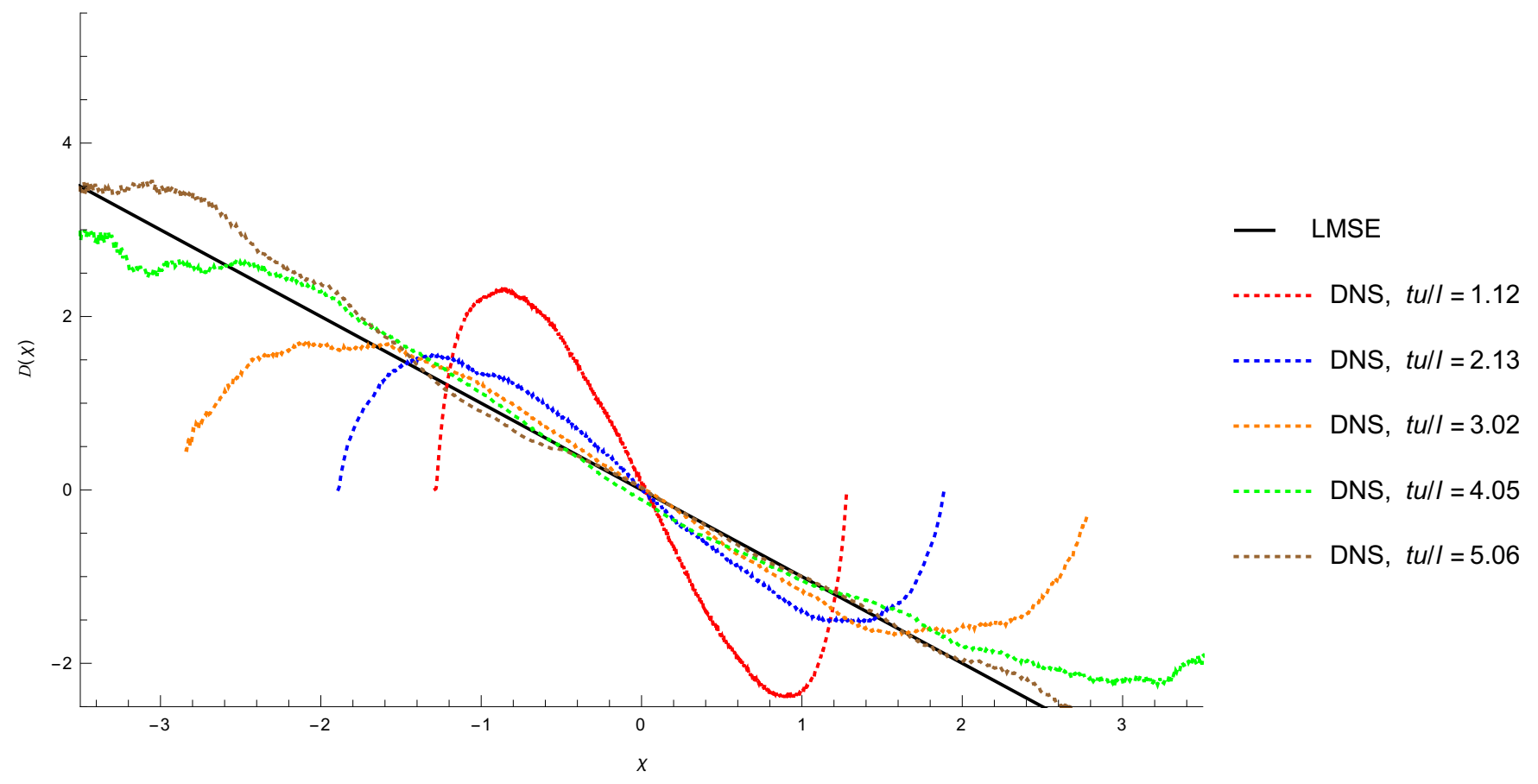

FIG. 2. Conditional diffusion. Symmetric case.

is made.

In the next section, a generalization of the binomial Langevin model ${ }^{35}$ based on a quasi-stationary closure of $E(\chi, t)$ will be explored.
V. QUASI-STATIONARY MONTECARLO MODEL

A MonteCarlo method which uses a combination of LMSE for conditional diffusion and a quadratic estimation of conditional dissipation fails to modify the given values of skewness and kurtosis. In effect, LMSE tends to preserve statistical moments of order higher than two; whereas a combination of a 


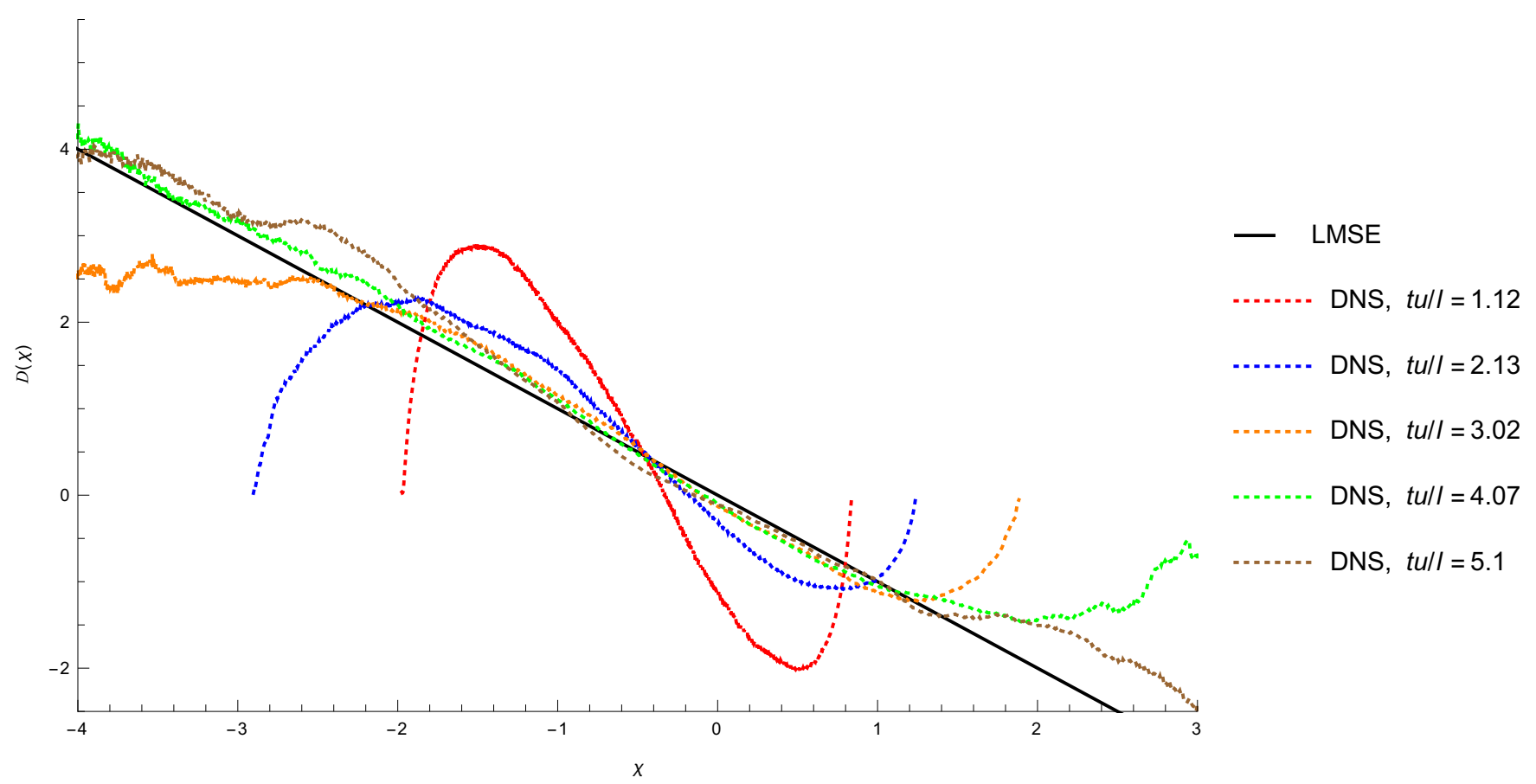

FIG. 3. Conditional diffusion. Asymmetric case.

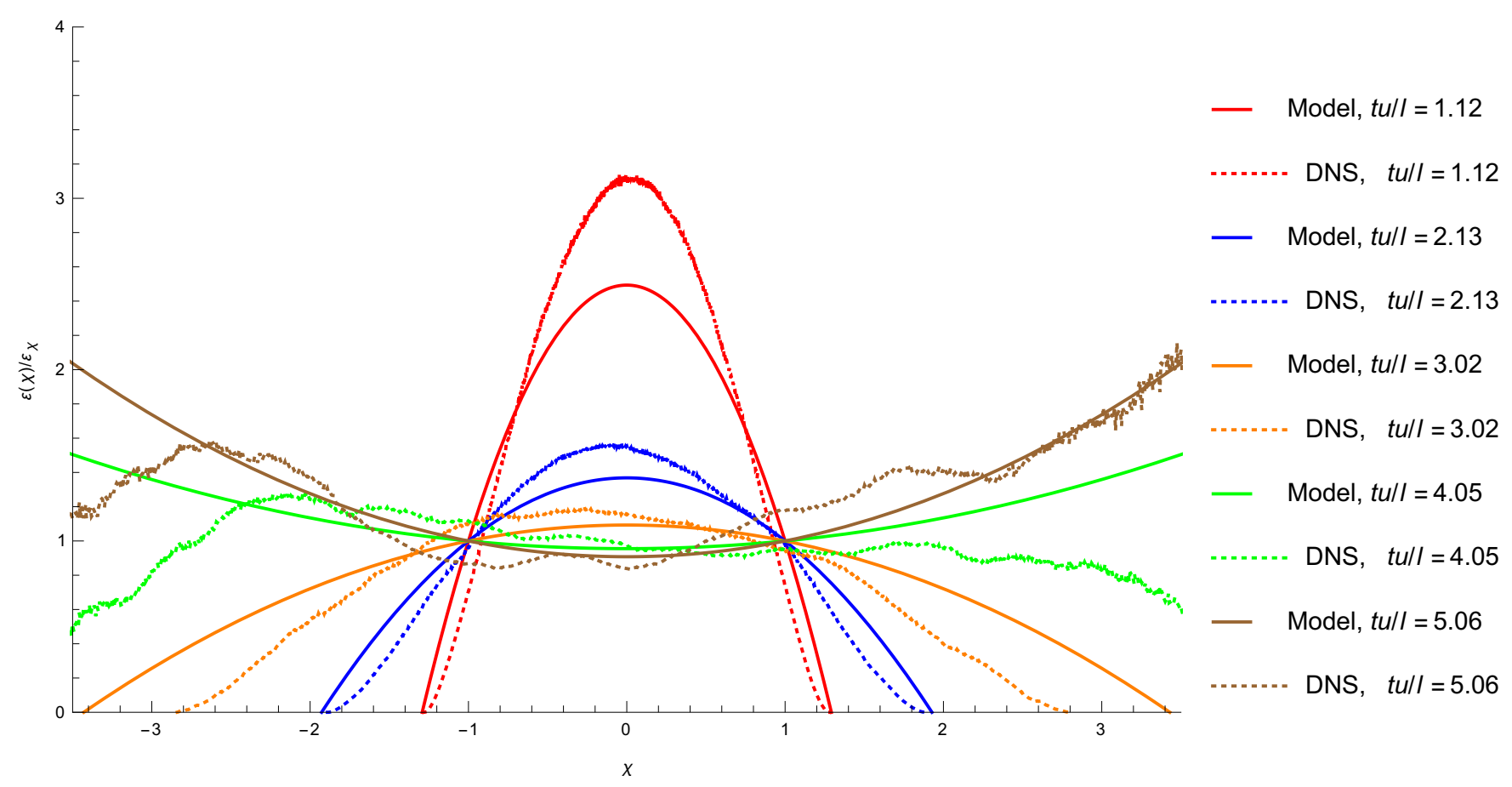

FIG. 4. Conditional dissipation. Symmetric case.

quadratic $E(\chi, t)$ and a linear $D(\chi, t)$ tends to relax any given initial PDF to an asymptotic state with the given values of skewness and kurtosis. However, it could be used when information about the final values of skewness and kurtosis is known. Such information is not usually available, but if provided, one can develop a MonteCarlo process which, from any given initial condition, will converge towards a PDF with the given values of skewness and kurtosis.

Work will be done in non-normalized fluctuation stochastic 


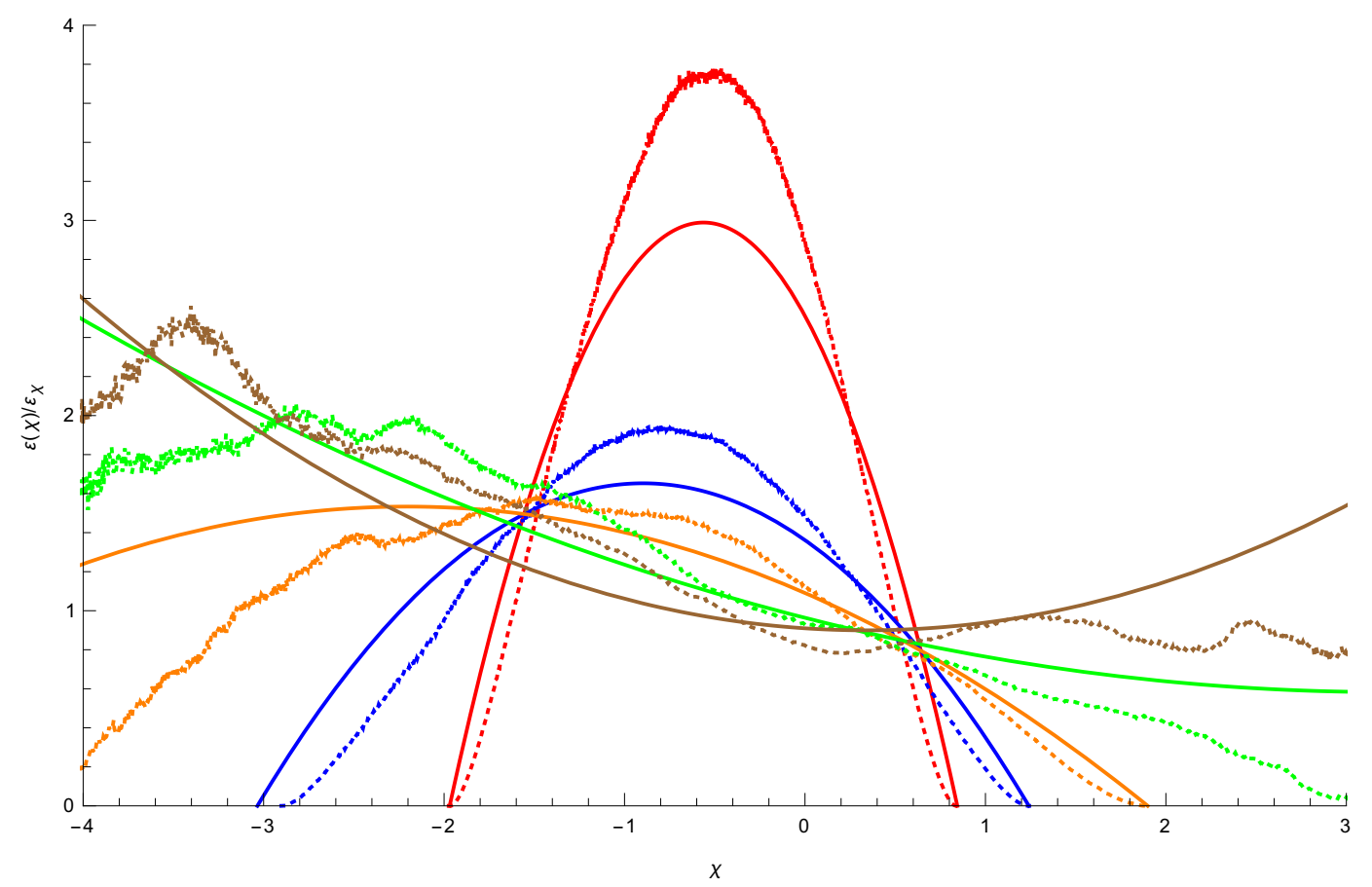

Model, $t u / I=1.12$

DNS, $\quad t u / l=1.12$

Model, $t u / I=2.13$

DNS, $\quad t u / l=2.13$

Model, $t u / I=3.02$

DNS, $\quad t u / l=3.02$

Model, $t u / I=4.07$

DNS, $\quad t u / l=4.07$

Model, $t u / l=5.1$

DNS, $\quad t u / l=5.1$

FIG. 5. Conditional dissipation. Asymmetric case.
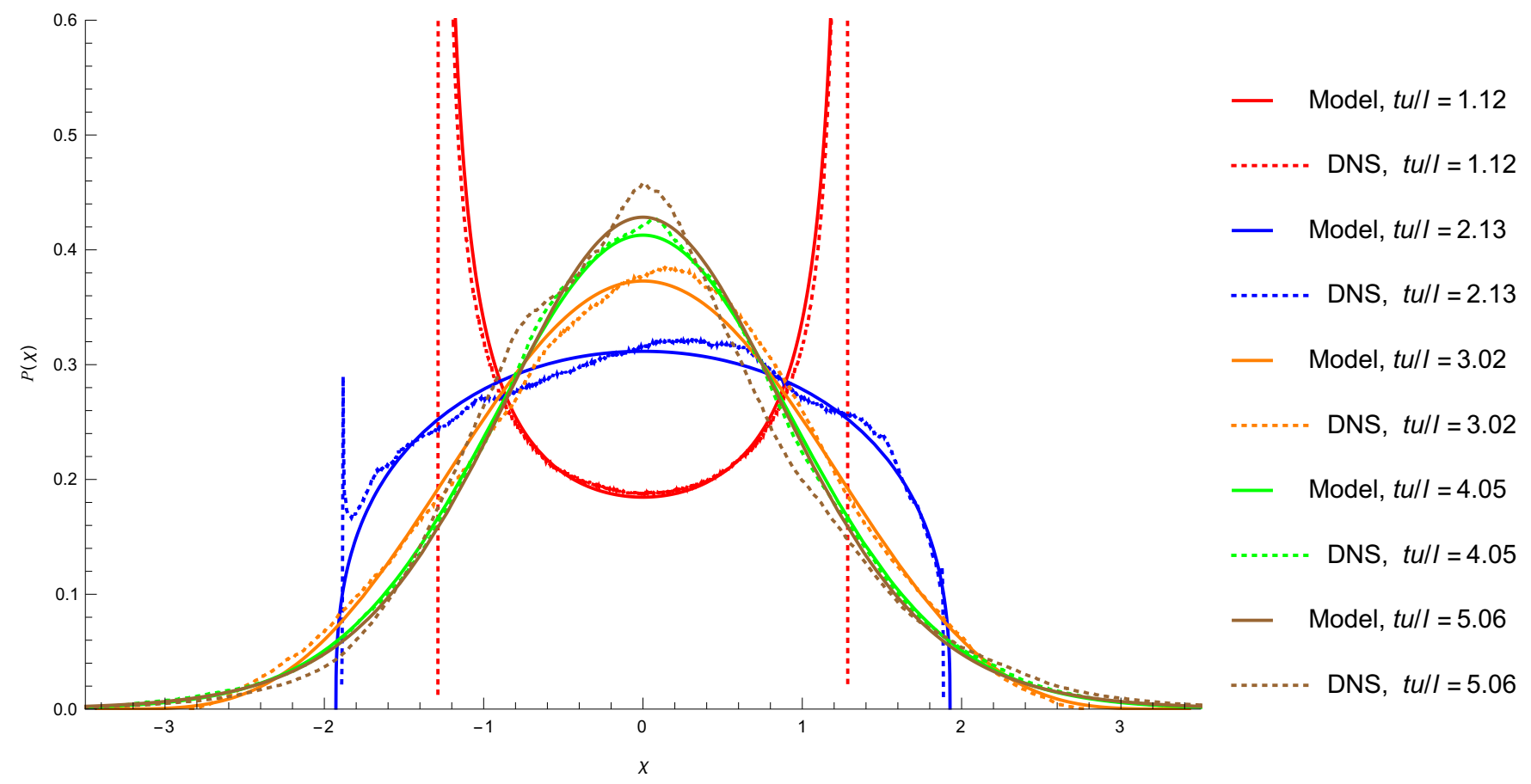

FIG. 6. Scalar PDF. Symmetric case.

variables $\phi=\sigma_{y} \chi . E(\phi, t)$ will be given by

$$
E(\phi, t)=\left\langle\alpha|\nabla Y|^{2}\right\rangle \frac{1+\left(C_{1} / \sigma_{y}\right) \phi+\left(C_{2} / \sigma_{y}^{2}\right) \phi^{2}}{1+C_{2}} .
$$

It is straightforward to check that $E(\phi, t)$ has been normalized so that $\langle E(\phi, t)\rangle=\left\langle\alpha|\nabla Y|^{2}\right\rangle=\sigma_{y}^{2}\left\langle\varepsilon_{X}\right\rangle$.
The binomial Langevin model ${ }^{35}$ is given by the stochastic equation

$$
d \phi=-\left\langle\varepsilon_{X}\right\rangle(1+k) \phi d t+\left(2 k\left\langle\alpha|\nabla Y|^{2}\right\rangle f(\phi)\right)^{1 / 2} d W_{t}
$$

where $d W_{t}$ is a Wiener process and $f(\phi)$ is a second order polynomial in $\phi$ with unity mean value, $\langle f(\phi)\rangle=1$, which be- 


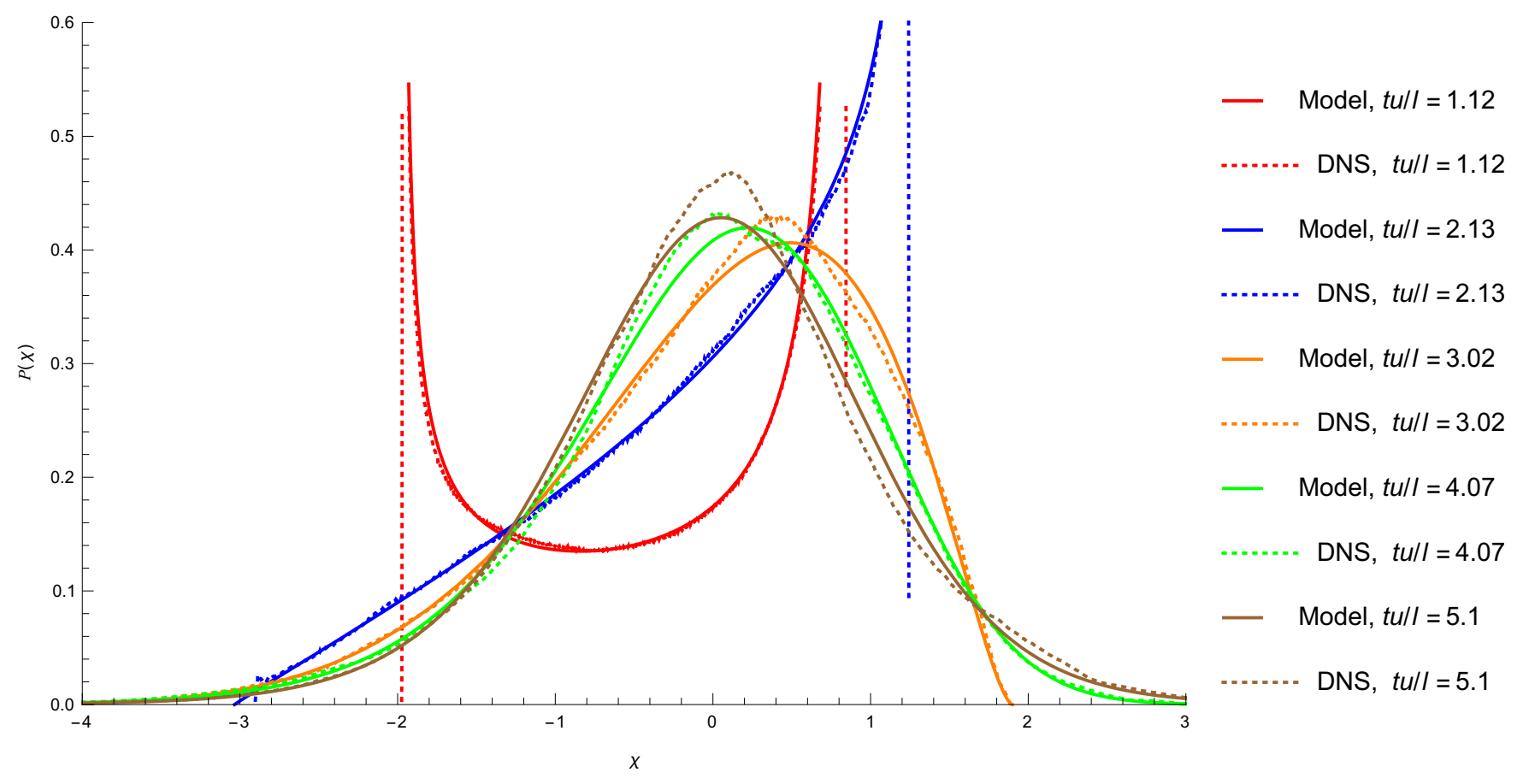

FIG. 7. Scalar PDF. Asymmetric case.

comes zero at the physical boundaries of scalar fluctuations. For instance: $\phi \in[-\langle Y\rangle, 1-\langle Y\rangle]$ when $Y \in[0,1]$ is a concentration. In such a situation, $Y \in[0,1]$, the function $f(\phi)$ is given by

$$
\begin{aligned}
f(\phi) & =\frac{\phi_{\min } \phi_{\max }-\left(\phi_{\min }+\phi_{\max }\right) \phi+\phi^{2}}{\phi_{\min } \phi_{\max }+\sigma_{y}^{2}} \\
& =\frac{\langle Y\rangle(\langle Y\rangle-1)+(2\langle Y\rangle-1) \phi+\phi^{2}}{\langle Y\rangle(\langle Y\rangle-1)+\sigma_{y}^{2}} .
\end{aligned}
$$

The binomial Langevin model, with a model constant $k=$ 0.7 , works well in situations where the expected asympotical statistics is Gaussian ${ }^{35}$ and has the nice property of corresponding to a Fokker-Planck process with zero diffusion at the physical boundaries of the domain. It means that it is easy to ensure the physical boundedness of the stochastic variables since a Wiener process leading towards a boundary is more and more reduced as it approaches the boundary. However, the binomial Langevin model is not good in providing asymptotic values of statistical moments which may be not near Gaussian ones. The reason being that, as variance gets reduced, most of the probability is concentrated close to the mean value where $f(\phi)$ is almost flat as a function of $\phi$. A perfect Gaussian would be obtained if $f(\phi)$ were constant.

The idea in order to model situations where long time values of skewness and kurtosis are known and they are not Gaussian is to keep eq. (31) and modify the function $f(\phi)$ with the limitation that it has unity mean value. According to previous results, such a function could be based on eq. (30)

$$
f(\phi)=\frac{1+\left(C_{1} / \sigma_{y}\right) \phi+\left(C_{2} / \sigma_{y}^{2}\right) \phi^{2}}{1+C_{2}} .
$$

However, such a function has the problem that it does not guarantee boundedness and a truncation of scalar values which move out of the physical boundaries is needed. This problem is specially important when the PDF has spikes close to the boundaries as it happens in the initial steps of evolution from a double delta.

As a proof of concept, in this paper, $f(\phi)$ was taken as the binomial version, eq. (32), for kurtosis less than its initial value plus 0.65 and as the quasi-stationary version, eq. (33), with the known values $S=-0.15$ and $F=3.78$ for the asymmetric scalar and $S=0$ and $F=3.76$ for the symmetric one. The limiting value $F=F_{0}+0.65$ was chosen because the value of the PDF close to the physical boundaries had already become almost zero by then and truncation errors were small. An interpolation between both functions for values of kurtosis close to $F_{0}+0.65$ would have provided a smoother transition, but it was not tried to keep the model simple. When there is high probability close to the boundaries, the model behaves as the binomial Langevin with truncation error under control and, when the probability close to the boundaries becomes very low, the model behaves as a MonteCarlo process whose asymptotical statistics tend towards the given skewness and kurtosis.

In figure 8 , results of the variance of the non-normalized fluctuation scalar field, $Y-\langle Y\rangle=\phi$, are given for DNS and for the model with two different samplings: $N=65536$ and $N=1024$. Data are provided for the symmetric and the asymmetric scalar. No big errors due to truncation appear.

In figure 9, results of the skewness of the asymmetric scalar field are given for DNS and for the model with two different samplings: $N=65536$ and $N=1024$. Results are not impres- 


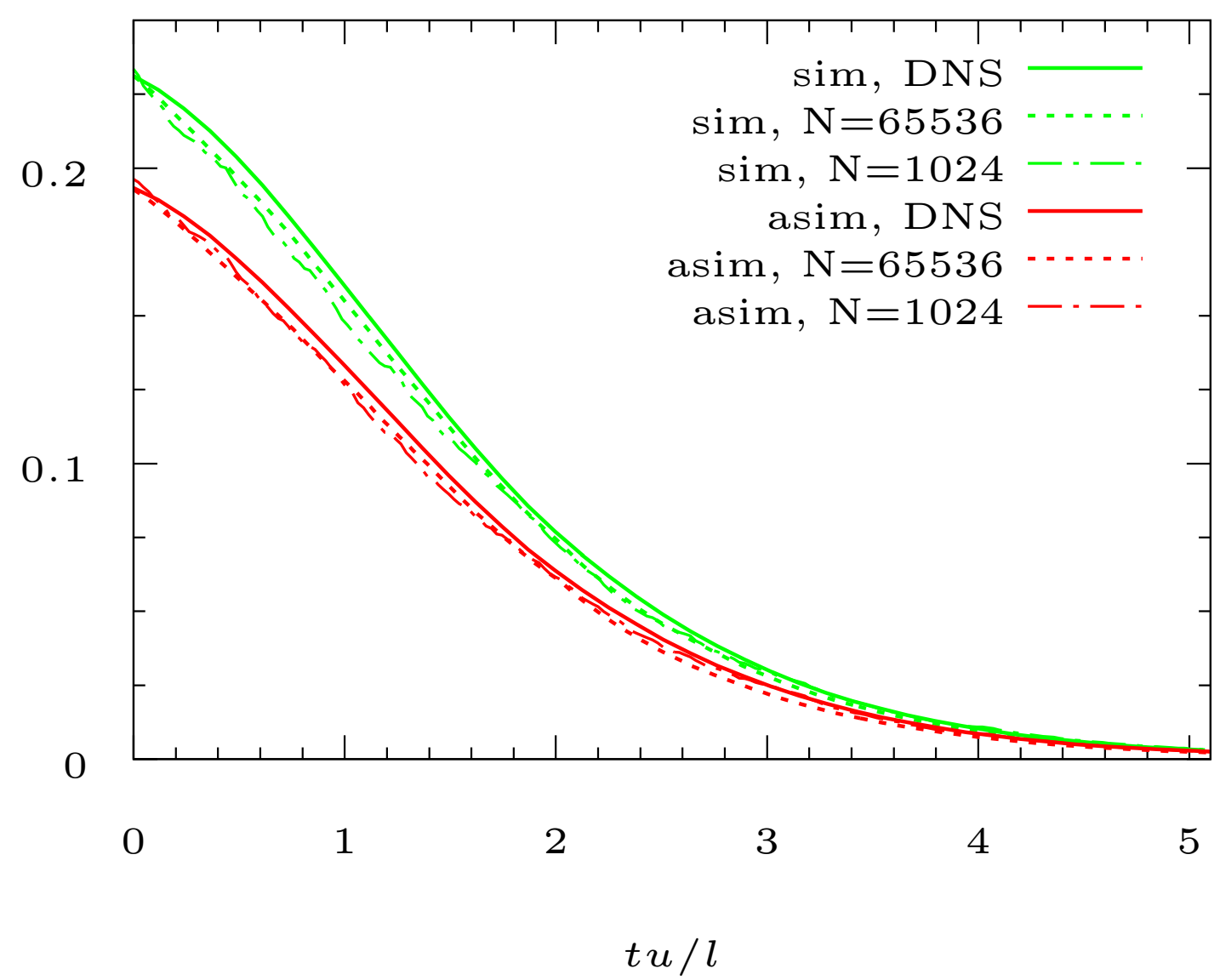

FIG. 8. Scalar fluctuation variance. Symmetric (green) and asymmetric (red) cases. Comparison of DNS data with the model proposed with two different samplings: $N=65536$ and $N=1024$ MonteCarlo particles.

sive, yet the evolution trend is captured.

In figure 10, results of the kurtosis are given for DNS and for the model with two different samplings: $N=65536$ and $N=1024$. Data are provided for the symmetric and the asymmetric scalar. Results look much better than those of skewness as it could be expected since even-order statistical moments comply with quasi-stationarity better than odd-order statistical moments.

\section{DISCUSSION AND CONCLUSIONS}

A merit of approximating the conditional dissipation by a quadratic polynomial is that it captures accurately the PDF behavior in a wide scalar range where the probability is high making an analytic solution possible. Obviously, the approximation fails near the scalar bounds (the tails of the PDF), the conditional diffusion being specially at odds with DNS results there. In previous research ${ }^{7}$, the conditional diffusion was not shown and its discrepancy with the numerical prediction was not investigated.

As the PDF spans over an unbounded scalar range the an- alytic solution disagrees with the numerical predictions. This unboundedness is a reflection of the insufficiency of a second order polynomial to approximate the conditional dissipation. This failure has been previously associated to models not reflecting the migration of scalar bounds ${ }^{14}$. Obviously, conditional dissipation must tend to zero at the extremal values where the first spatial derivative vanish. One of the problems is, that after some mixing evolution time, the initially convex conditional dissipation becomes concave. A fourth order polynomial is needed for a concave conditional dissipation in the high probability region to bend and vanish as the real scalar bounds are approached, whereas a quadratic approximation causes the conditional dissipation to grow with no limits away from the scalar mean.

The range of probable scalar values is realistically bounded using a fourth order polynomial for the conditional dissipation rate. The latter implies including fifth and sixth order scalar central moments, the super-skewness and the superkurtosis, to calculate the two extra coefficients of the polynomial. These statistical parameters do determine the behavior of the PDF tails, in the neighborhood of the two finite scalar bounds. Unfortunately, no analytic solutions are possible with 


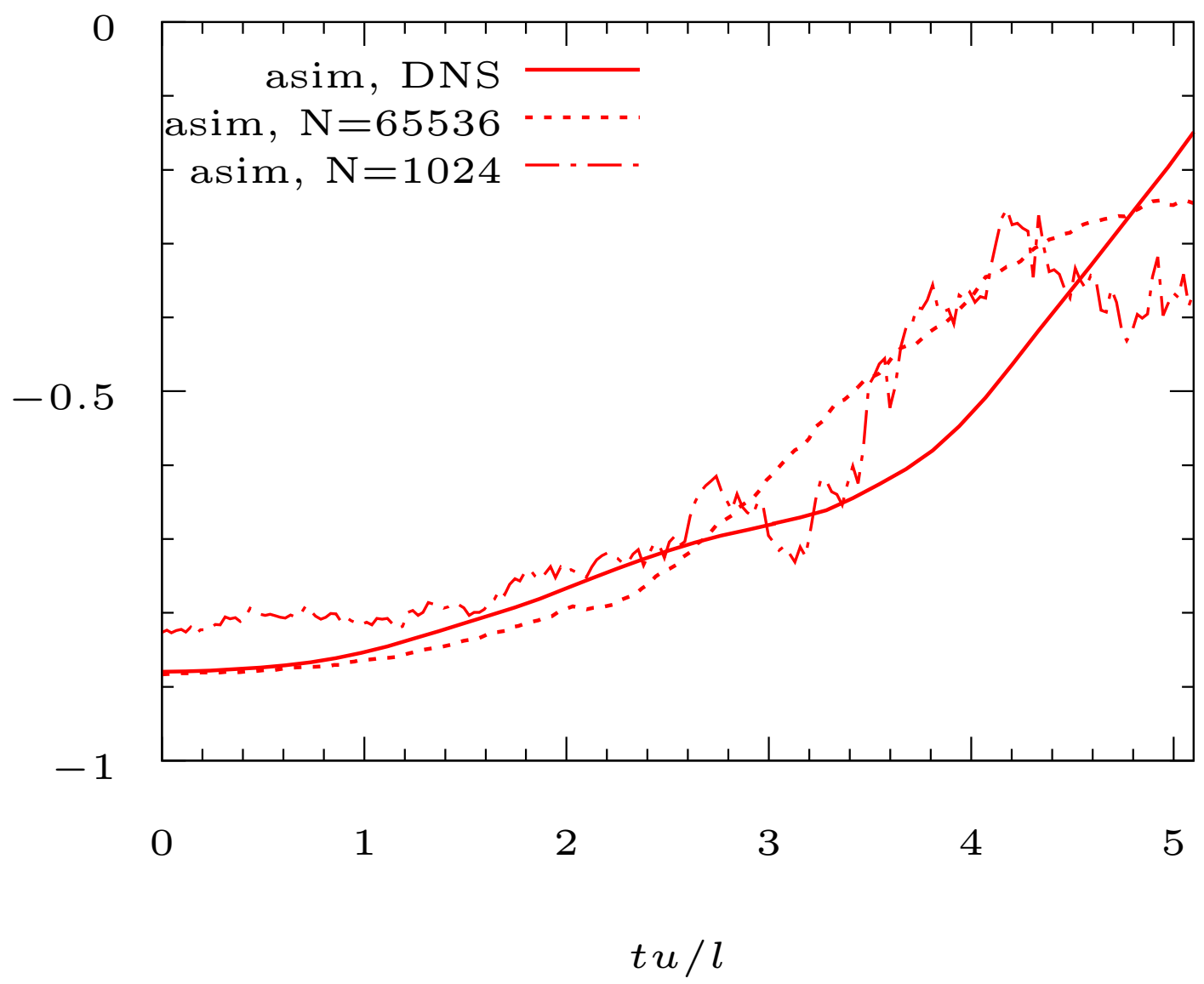

FIG. 9. Scalar skewness. Asymmetric case. Comparison of DNS data with the model proposed with two different samplings: $N=65536$ and $N=1024$ MonteCarlo particles.

a fourth order approximation of the conditional dissipation, apart from the polynomial coefficients.

A modification of the binomial Langevin model whose asymptotic PDF may show a non-Gaussian behavior has been developed. Nevertheless, this model would only be useful if information about the final values of skewness and kurtosis was previously available. The model drives the PDF towards a state characterized by given skewness and kurtosis, yet it cannot predict those given values.

As future work lines, one might consider: i) the use of third and fourth order polynomial approximations for the conditional diffusion and dissipation, respectively, as they would guarantee a better behavior of these variables at the scalar bounds; ii) An inert scalar field in decaying homogeneous turbulence; iii) longer simulation times to check whether some trend towards PDF Gaussian shapes finally emerges; iv) the effect of nonlinear chemical source terms with constant and variable densities; and v) the quasi-stationary properties of scalar gradients.

\section{ACKNOWLEDGMENTS}

The authors acknowledge the research of E.E. O'Brien.

${ }^{1}$ Ya. G. Sinai and V. Yakhot, "Limiting probability distributions of a passive scalar in a random velocity field," Phys. Rev. Lett. 63, 1962 (1989).

${ }^{2}$ V. Yakhot, S.A. Orszag, S. Balachandar, E. Jackson, Z.S. She and L. Sirovich, "Phenomenological theory of probability distributions in turbulence," J. Sci. Comput. 5, 199 (1990).

${ }^{3}$ V. Eswaran and S.B. Pope, "Direct numerical simulations of the turbulent mixing of a passive scalar," Phys. Fluids 31, 506 (1988).

${ }^{4}$ E.S.C. Ching, "Probability densities of turbulent temperature fluctuations," Phys. Rev. Lett. 70, 283 (1993).

${ }^{5}$ E.E. O'Brien and T.L. Jiang, "The conditional dissipation rate of an initially binary scalar in homogeneous turbulence," Phys. Fluids A 3, 312 (1991).

${ }^{6} \mathrm{~F}$. Gao, "Mapping closure and non-Gaussianity of the scalar probability density functions in isotropic turbulence," Phys. Fluids A 3, 2438 (1991).

${ }^{7}$ L. Valiño, C. Dopazo and J. Ros, "Quasistationary probability density functions in the turbulent mixing of a scalar field," Phys. Rev. Lett. 72, 3518 (1994).

${ }^{8}$ F.A. Jaberi, R.S. Miller and P. Givi, "Conditional Statistics in Turbulent Scalar Mixing and Reaction," AIChE J. 42 (4), 1149-1152 (1996).

${ }^{9}$ H.L. Toor, "Mass Transfer in Dilute Turbulent and Nonturbulent Systems with Rapid Irreversible Reactions and Equal Diffusivities," AIChE J. 8, 70 (1962). 


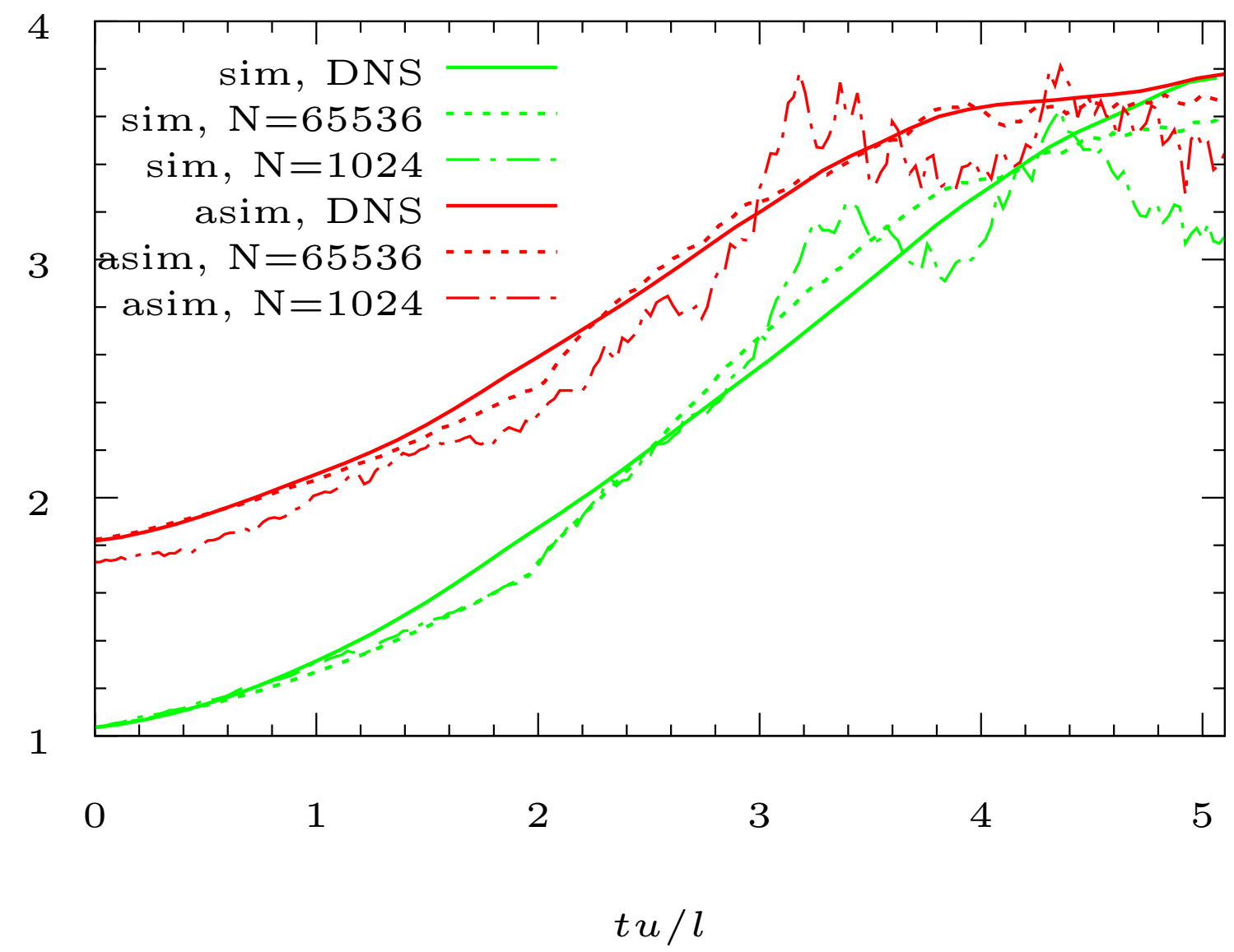

FIG. 10. Scalar kurtosis. Symmetric (green) and asymmetric (red) cases. Comparison of DNS data with the model proposed with two different samplings: $N=65536$ and $N=1024$ MonteCarlo particles.

${ }^{10}$ C. Dopazo, "Recent development in PDF methods," in Turbulent Reacting Flows, Eds. P.A. Libby and F.A. Williams, 375-474, Academic Press (1994).

${ }^{11}$ E.Y. Edgeworth, "On the representation of statistical frequency by a series," J. Royal Statist. Soc. A 70, 102 (1907).

${ }^{12}$ N.L. Johnson. N. L., "Systems of frequency curves generated by methods of translation," Biometrika 36, 149 (1949).

${ }^{13}$ R.H. Kraichnan, Closures for probability distributions, Bull. Amer. Phys. Soc. 34, 2298 (1989).

${ }^{14}$ R.S Miller, S.H. Frankel, C.K. Madnia and P. Givi, Johnson-Edgeworth, "Translation Probability Modelling of Binary Scalar Mixing in Turbulent Flows," Combust. Sci. Tech. 91, 21 (1993).

${ }^{15} \mathrm{~J}$. Sukhatme, "Probability density functions of decaying passive scalars in periodic domains: An application of Sinai-Yakhot theory," Phys. Rev. E 69, 056302 (2004).

${ }^{16}$ A.N. Kolmogorov, "A refinement of previous hypotheses concerning the local structure of turbulence in a viscous incompressible fluid at high Reynolds number," J. Fluid Mech. 13, 82 (1962).

${ }^{17}$ A.Y. Kuo and S.Corrsin, "Experiments on internal intermittency and finestructure distribution functions in fully turbulent fluid," J. Fluid Mech. 50, 285 (1971).

${ }^{18}$ R.A. Antonia and K.R. Sreenivasan, "Log-normality of temperature dissipation in a turbulent boundary layer," Phys. Fluids 20, 180-1804 (1977).

${ }^{19}$ R.A. Antonia, E.J. Hopfinger, Y. Gagne and F. Anselmet, "Temperature structure functions in turbulent shear flows," Phys. Rev. A 30, 2704-2707 (1984).
${ }^{20}$ R.S. Miller, F.A. Jaberi, C.K. Madnia and P. Givi, "The structure and the small-scale intermittency of passive scalars in homogeneous turbulence," $J$. Sci. Comput. 10, 151-180 (1995).

${ }^{21}$ S.B. Pope, "Turbulent Flows," Cambridge University Press, Cambridge (2000).

${ }^{22} \mathrm{C}$. Cerutti and C. Meneveau "Statistics of filtered velocity in grid and wake turbulence," Phys. Fluids 12, 1143-1165 (2000).

${ }^{23}$ S. Cerutti, C. Meneveau and O. M. Knio, "Spectral and hyper eddy viscosity in high-reynolds-number turbulence," J. Fluid Mech. 421, 307-338 (2000).

${ }^{24}$ S. Orszag and G. Patterson, "Numerical simulation of turbulence: statistical models and turbulence," Lecture Notes in Physics 12, 127-147 (1972).

${ }^{25}$ R.S. Rogallo and P. Moin, "Numerical simulations of turbulent flows," Ann. Review Fluid Mech. 16, 99-137 (1984).

${ }^{26}$ S. Cox and P. Matthews, "Exponential time differencing for stiff systems," J.Comp. Physics 176, 430-455 (2002).

${ }^{27}$ A. Kassam and L. Trefethen, "Fourth-order time stepping for stiff pdes," SIAM J. Sci. Comp. 26, 1214-1233 (2005).

${ }^{28}$ V. Eswaran and S.B. Pope, "An examination of forcing in direct numerical simulations of turbulence," Computers and Fluids 16, 257-278 (1988).

${ }^{29} \mathrm{~K}$. Alvelius, "Random forcing of three-dimensional homogeneous turbulence," Phys. Fluids 11, 1880-1889 (1999).

${ }^{30}$ J. Jiménez, A.A. Wray, P.G. Saffman and R.S. Rogallo, "The structure of intense vorticity in isotropic turbulence," J. Fluid Mech. 255, 65-90 (1993).

${ }^{31} \mathrm{H}$. Tennekes and J.L. Lumley, "A first course in turbulence," MIT Press, ISBN: 978-0262200196 (1972)

${ }^{32}$ Z. Warhaft, "Passive scalars in turbulent flows," Ann. Rev. Fluid Mech. 32, 
203-240 (2000).

${ }^{33}$ F.A. Jaberi, R.S. Miller, C.K. Madnia and P. Givi, ”Non-Gaussian scalar statistics in homogeneous turbulence," J. Fluid Mech. 313, 241-282 (1996).

${ }^{34}$ H. Chen, S. Chen and R.H. Kraichnan, "Probability distribution of a stochastically advected scalar field,", Phys. Rev. Lett. 63, 2657 (1989).
${ }^{35}$ L. Valiño and C. Dopazo, "A binomial Langevin model for turbulent mixing", Phys. Fluids A 3, 3034 (1991).

${ }^{36}$ R.O. Fox, "The Fokker-Planck closure for turbulent mixing: Passive scalars", Phys. Fluids A 4, 1230 (1992). 